\title{
Developing Two-Armed Robot Assistant: Intelligent Tutoring Skills Based System
}

\author{
Ivan Vladimirovich Krechetov ${ }^{1 *}$, Arkadiy Alekseyevich Skvortsov ${ }^{1}$ \\ and Pavel Sergeyevich Lavrikov ${ }^{2}$ \\ 'Office of Scientific Research and Development, Moscow State University of Mechanical Engineering (MAMI), \\ krechetov_ivan@mail.ru,skvortsov@mami.ru \\ 2RU.Robotics, Rubtsov per, 13, Moscow, Russia, pavel_lavrikov@rurobotics.com
}

\begin{abstract}
Background/Objectives: The objective of this study is to develop constructive and firmware solutions to create universal robot that would perform different simple tasks and replace human labor in remote autonomous operations. Methods: Specific features of the suggested learning method are represented by the possibilities to copy and exchange skills between different robots including those that possess different kinematic schemes. This becomes possible due to the adaptive control over the manipulator links in line with the current configuration of the effective area. Findings: The study suggests an approach to controlling robots as they perform operations under different conditions including those that are not predetermined by the working area and in cases with obstacles based on earlier obtained knowledge (skills) and according to the universal model of control over performing one particular acquired practice (complex operation with objects) in undetermined environment as a combination of elementary actions performed in real-time mode revealing the capability of adapting to the changing conditions of the environment. Applications/Improvements: The results of the study can be applied to constructive, hardware and software solutions in the process of developing a universal robot to replace manual labor in remote autonomous operations.
\end{abstract}

Keywords: Collaborative Robot, Human-Robot Collaboration, Manipulator Kinematics, Robot Learning, Robot Tasks, Skills, Two-Armed Robot

\section{Introduction}

Introduction of robotics in small businesses improves the efficiency and quality of the production process. Due to this fact it is often required that small enterprises should be upgraded to conform to the changes in the production lines and that the robots should be adjusted accordingly. However, this reconfiguration of robots is ought to be simple enough to be done by a common production worker and it ought not require any special knowledge or programming skills in this subject area.

Over the last several years the product lines of many industrial robot manufacturers, including such big market players as ABB YuMin, Fanuc (CR-35iA) KUKA and Yaskawa Motoman (HC10) have been enhanced by small manipulators and two-armed robots called "collaborative". These robots feature human-like size, kinematic schemes and developed forces. These robots are employed for human-robot collaboration or for replacing human personnel altogether.

Thus, the situation on the market of collaborative robots and the conditions of scientific and technical solutions for controlling the robots as they perform complex tasks confirm the current importance of the subject which is aimed at establishing internal capacities for developing domestic competitive robotic systems.

\section{Concept Headings}

\subsection{Application of Collaborative Robots}

The existing studies in the sphere of creating the control systems for manipulator robots that would ensure safe working conditions in the process of human-robot collaboration aim to solve the problems of the control over the developed forces and those of the planned trajectories of multilink manipulators based on imitating

${ }^{*}$ Author for correspondence 
the movements of the operator. However, these robots can only be used if their environment and their effective area have been predetermined and preconfigured in advance which restricts their use in cases when the operating conditions are changing and when each robot has to be reconfigured individually for the relevant modes; moreover, such robots are, as a rule, static and cannot be relocated. To insure safe operations in the undetermined environment and in dynamically changing situations when static and movable obstacles can emerge, the investigations have to be carried out to develop new constructive, hardware and software solutions that would enable robots to copy and to exchange the skills required for performing the operations automatically without the need to be programmed individually.

Principle advantages of the collaborative robots are represented by their ability to operate with a human worker, by their capability to co-operate with people in close contact without the need to fence their effective area. These advantages help save the available production space.

A collaborative robot operates either together with or instead of man providing a substitute in cases where labor is associated with intensive physical loads or danger making it unnecessary for a human worker to lift heavy objects or to handle toxic materials thus ensuring safer working environment and reducing overall labor intensity.

The major advantages of such robots are listed below:

easy introduction into industrial environment (universal nature; compact, human-like sizes; adjustability);

safe cooperation with a man; no fences;

saving the available working space because no fencing is required;

light and compact design can be removed easily and occupy small areas;

replacing a human worker (or providing assistance to the personnel) in performing dangerous and/or labor intensive tasks;

efficiency in performing continuous operations and easy reconfigurations for other purposes;

The process automation systems can use collaborative robots for operations as follows:

Transporting and removing parts and work-pieces;

Assembly operations;

Conveyor charging;
Machine tools operations;

Hand tools operations;

Product classification;

Packaging.

The framework of robot operating system (ROS) $)^{-8}$ is becoming widely popular among the designers of robotic systems in different application areas

ROS is an aggregate of services that ensure the functional features as follows:

1) HAL, hardware abstraction layer that makes it possible, by means of the same methods and interaction protocols, to control the operations of the units no matter which architecture of the computation module they use;

2) Transportation of messages between the processes, the principle peculiar feature of ROS that implies the possibility to transmit one and the same data (messages) between different configurations of the sources (publisher) and consumer (subscriber) (star, series, etc.);

3) Low level units control: extensive support of the drivers of base operating system;

4) Supporting and performing a large number of functions: widely applied by the developers, ROS has actually become a standard in robotics. Over the years of its development ROS project has acquired a huge number of different functions and methods in the theory of signal control and processing, in the sphere of computer vision and in solving the problems of reverse kinematics, etc. Thereat, due to its hardware abstraction this set of functions can be used for implementing other new projects. The official site provides full package of documents on the developed software modules that also contain the information on application areas, requirements to the slave modules, manuals for installation, customization and operation.

5) Process graph representation: in all, the operating system based on ROS can activate a large number of functions with great amount of messages sent between them. The graph input and output module can vividly represent these interactions thus making it possible to identify the redundant dependencies in the course of the system performance analysis and to optimize the structure of the involved modules.

6) Graphic module set enables visualization and interface for controlling the current state of the system supporting the wide range of typical data:

a) Time schedules (the data are represented by the elements of the message structure); 
b) Slider bars for parameter control that can affect the system in manual operation mode;

c) $3 \mathrm{D}$ model provides rendering and visualization of the current locations of the units and assemblies of robot as interconnections between the elements of 3D-model;

d) Displaying images makes it possible to watch video stream in computer vision systems;

e) Displaying texts and symbols is a traditional method of representing the information on the current parameter values which is widely applied in debugging modes.

7) Matrix Laboratory (MATLAB) supports.

8) Multisite operations: each software module (function) can be launched at a separate (several) computation module which is integrated into the common local network.

ROS is not a real-time operating system; however, in developing the core of this system the data exchange protocols between publishers and subscribers are of paramount importance. The developer can adjust the parameters of the receiving and sending buffers in most optimal manner depending on the intensity of the data exchange and on the requirements to the performance of the computation module.

For controlling the multilink manipulators, for planning the movement trajectories and for modeling the contact interactions two additional packages can be used in addition to ROS:

1) Gazebo - simulation and visualization of physical processes;

2) MoveIt! - A set of functions for calculating trajectories and for controlling the movements of multilink manipulators.

\subsection{Collaborative Robot Programming System}

The most important part of the robotic system which makes its own contribution into the efficiency of the system performance in general is represented by the tools for programming or teaching the robot. Inasmuch as the programming is done by a human operator, all leading manufacturers of collaborative robots try to reduce the human factor and to make this process as simple as possible so that it could be easily performed even by lowqualified professionals.

Classical method of robot programming is to set the trajectory manually applying specialized software; thereat, to control the operations of the robot, this would require creating a virtual model of the environment in which the robot is supposed to operate. The programming process in very laborious and time consuming, it has to involve highly-qualified personnel and complete simulation of the whole system. Any day-to-day adjustments to the programs are difficult; therefore, employment of robots can only be justified under the conditions of the largescale production.

The following trends of programming modern robots have been identified:

1) Teaching from demonstration ${ }^{-15}$

2) Behavioral control and skills, generalization of operations ${ }^{20-32}$

\subsection{Teaching from Ocular Demonstration}

As an alternative to conventional programming many methods have been suggested and one of them is represented by learning or programming through demonstration which eliminates the difficulties associated with the process. This concept implies training the robot to move by manual demonstration of the tasks and it represents the synthesis of the techniques of several disciplines, including machine learning, computer vision, electric motor control and interactions with the robot by means of demonstration for the purposes of its programming and control.

Thus, in the services sector where there is a great demand for the robots that would interact with human beings the key driver for enhancing robot applications in everyday life may be represented by the opportunities for the intuitive programming of these robots by the final users. In this regard, considerable scientific attention is paid to the method of demonstration-based teaching according to which the robot can learn how to perform the task using some examples.

By contrast to the conventional robot programming, demonstration-based programming makes it possible for the robot to learn a part of the movement or to perform the task completely by observing one or several demonstrations of the movements or actions performed by a man.

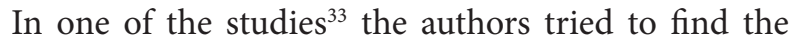
differences in training three different robots using the demonstrations performed by common people that were not experts in this area. The study included the methods based on Behavior Networks, Interactive Reinforcement Learning, and Confidence Based Autonomy. 
Over the last several years there was a considerable increase in the number of the studies that were dedicated to robot learning from demonstration; however, the results were evaluated without taking into account the opinions of the users, as the learning process was founded on algorithmic models and no users were involved. Consequently, this study described the experience of the non-expert users (common users) interacting with robots.

Replacing standardized programming by interactive learning from demonstration enhances the functional capabilities of the robots in providing services to people. Based on human demonstrations the system formulates the behavioral pattern of the new robot. Moreover, developing the intuitive programming method that would be comprehensible to an unqualified and untrained person is an important objective of this investigation.

Another study suggests a scheme for kinesthetic action-based behavioral teaching for the torquecontrolled robots.

Over several decades the researchers have been trying to solve the complex tasks that would make it possible to use robots in everyday life in the same way as people use computers now. If robots can cooperate with people then greater number of tasks will be performed easily, fast and safely. This objective can be achieved if the robots are given the opportunities to learn from the actions demonstrated by people and to develop based on their own experience.

One of the natural ways to solve this task is represented by learning from demonstration when the robot obtains knowledge from man as examples of behavior. Other methods are based on developing learning algorithms for collecting data on the demonstrations performed by the teacher applying optical or computer vision systems for programming the trajectory. Besides, new patterns of control are implemented that are founded on the modifications of the existing methods and take into account the new opportunities provided by the alternate actions featured by modern manipulator robots thus making it possible to perform complex tasks.

Today action-based control and kinesthetic information transfer are the most important tasks that attract very much attention in the course of studying physical interactions between human beings and robots.

Some authors 5 suggest an approach to interactive learning with a multilevel mechanism for training the interaction policies that would prevent collisions in dynamically changing environment. Instead of developing complicated rules, applying simulation and parameter setting procedures it is deemed best that the assistant robot should be taught its behavior through demonstrations; however, such methods have a number of limitations. It is generally believed that there should be a controller or teacher (expert) who would collect demonstrations. Then, assisted by active learning, the useful information is extracted from the demonstrations based on the criterion that would be applied further in the course of learning. However, there is a disadvantage to this approach, namely, the fact that this criterion is created by the experts themselves while, according to the approach that is expostulated in this study, the trainee would not require any information about the teacher; moreover, upon the initial demonstration he should ask why the current learning policy results in the choice between several options or several emergencies instead of the dialog.

\section{Method}

Many investigations in the sphere of learning from demonstration have been dedicated to the cases when robots are taught single strategy by demonstrating a simple task that has clear beginning and end. However, in cases with complex tasks which can hardly be modeled with only one strategy, this approach often fails. Therefore, here it is not the whole task that is taught, but the approach that includes structured demonstrations of a series of subtasks or skills which can be learned easily and which can be used later as component parts of other tasks.

Inasmuch as the demonstration of the task is performed uninterruptedly from start to finish, the procedure takes too long and it is rather difficult to split the task into component skills; this would also require knowledge of the kinematic properties of the robot, of the skills already learned and of the internally perceived notions. Besides, special expertise would be required to evaluate whether two segments can be considered similar in terms of different skills and also to select the level of the details the segmentation is to be broken down to. Another thing to say is that after some time manual maintenance of this set of skills would become tiresome.

The solution of the abovementioned problem is to implement automated segmentation process. For this purpose the system designed for leaning the tasks of ever increasing complexity based on the non-segmented and, probably, incomplete demonstrations of several tasks or skills should meet the key requirements as follows:

- The robot has to be capable of recognizing the 
repeated examples of skills and to apply them to the new conditions.

- The robot should perform segmentation without possessing a-prior knowledge about the number and the structure of the skills that form the task.

- The robot must identify a wide generic class of skills including manipulations with objects, gestures and intended actions.

- There should be opportunities for improving the abovementioned skill strategies in practice.

The principal feature of the developed assistant robots, or in other words collaborative robot is represented by its ability to co-operate with man, its capability to interact with human worker in close contact without the need to fence the effective area.

The collaborative robot can operate with or instead of the human worker substituting the one in cases where human labor is associated with intensive physical loads or danger saving a man from the need to lift objects or to work with toxic materials thus creating safer working conditions and reducing labor intensity.

Collaborative robots that feature human-like size and that can be trained easily will help a man successfully or even replace one in performing continuously repeated tasks.

Thus, the major advantages of collaborative robots are represented by such properties as follow below:

- simple introduction into the existing production processes (universality; compactness; human-like sizes; easy adjustability);

- safe human-robot cooperation without any fencing;

- efficient use of the available space because no safety fencing is needed;

- usually light and compact and thus easily removable and requiring small installation areas;

- can replace a man (or provide assistance to a man) in solving the problems associated with danger and/or intensive loads;

- efficient in performing continuously repeated operations and can be easily reconfigured for other purposes;

Based on this first stage analysis of the techniques of programming the manipulator for different types of operations the following methods can be distinguished:

\subsection{Traditional (Computer Numeric Control)}

Programming complex trajectories by presetting intermediate coordinates of the manipulator end-effectors with CAM packages. This method is widely applied in processing industries, machine cutting and industrial robotics.

\subsection{Kinesthetic}

The robot responds to the externally applied force and follows the movements of the operator's hand. This is the principle method for programming collaborative robots based on the trajectory demonstrations performed by the user.

\subsection{Observation-based learning}

With this type of learning the robot analyzes the scene observed by the computer vision system and identifies the context by correlating the operations and manipulations with objects. The robot can independently formulate the trajectories required for performing the relevant operations.

\section{Result}

One of the specific characteristics of collaborative robots is that the tools for programming or learning are integrated in the robot itself and the programming can be performed directly in the physically installed robot. There are different ways of implementing the robot training aids: controlling joysticks installed on the manipulators, torque sensor systems installed in the joints, virtual reality gloves, etc.

Replacing standardized programming with interactive learning, for example, with learning from demonstration enhances the functionality of the robots making them more useful for people. Based on the human demonstrations the system formulates the behavior of the new robot. Moreover, developing the intuitive and comprehensible programming method that could be applied by an unqualified and untrained person is an important objective of further investigations.

Analyzing the application areas of collaborative robots and systematizing the performed tasks it is possible to decompose complex manipulations with objects into more simple actions that can be taught as separate groups of tasks. Thus, the hierarchical scheme of the skill performance scenario can be represented as follows below:

1. Skill performance scenario

1.1 Object identification

1.2 Database search 
1.3 Reading possible (known) operations

1.4 Visualization of options as a list

1.5 Operation selection

1.5.1 Relocation of the end-effector between points A $->\mathrm{B}$

1.5.1.1 The area delimitated with tags

1.5.1.2 Standalone tags

1.5.2 Relocation A->B and adjustments

1.5.2.1 Placing along the line tightly

1.5.2.2 Placing along the line with a specified gap

1.5.2.3 Installing in the cells of the array with pitch $x, y$ accordingly and with dimensions of $\mathrm{M}^{\star} \mathrm{N}$

1.5.2.4 Placing in the box as matrix $\mathrm{M}^{\star} \mathrm{N}^{\star} \mathrm{JK}$

1.5.3 Linking two objects

1.5.3.1 Master part orientation

1.5.3.2 Slave part orientation

1.5.3.3 Connection contour

1.5.3.3.1 End plane of the part

1.5.3.3.2 End plane center

1.5.3.3.3 End plane dimensions $\left(R, A^{\star} A, A^{\star} B\right)$

1.5.3.4 Alignment of two parts

1.5.4 Transportation of the objects (by means of the installed table)

1.5.4.1 Placing the objects on the table for transportation

1.5.4.2 Removing the objects from the

1.6 Determining the configuration of effective area

1.6.1 Availability of the finish zone

1.6.2 Availability of the limiting line

1.6.3 Option with finish demonstration

1.7 Identifying the grasping plane on the surface of the object (?) -> Central point

1.8 Identifying the possibility to perform manipulations

1.8.1 Identifying the possibility to reach the object (solving reverse kinematics for the central point)

1.8.2 Calculating trajectory

1.8.3 No collisions with the environment

1.9 Relocation of the end-effector to grasp the object

1.10 Grasping the object

1.11 Grasp control

1.11.1 Visual

1.11.2 Based on torque sensors applying impedance control

1.12 Relocation of the end-effector to point $B$

1.13 Releasing the object

2 Robot learning system

2.1 Kinesthetic
2.1.1 Memorizing trajectory

2.1.1.1 Initial position and orientation of the manipulator end-effector

2.1.1.2 Final position and orientation of the manipulator end-effector

2.1.1.3 Speed (time) of relocation between the neighboring points of the trajectory

2.1.2 Operation at the point (current orientation and position of the end-effector)

2.1.2.1 Grasp the object (closing end-effector)

2.1.2.2 Release the object (opening end-effector)

2.2 Visual (skills system)

2.2.1 Determination of spatial coordinates of the object

2.2.2 Recognizing the object

2.2.2.1 Extracting special points

2.2.2.2 Formulating the object descriptors

2.2.2.3 Recording the descriptors into the database

2.2.3 Identification of the object with the associated operation (trajectory)

2.2.4 Recording the skill into the database

The most important tasks that have to be solved by the computer vision system of the robot in order to grasp the objects are represented by object detection, object location recognition and estimation. Object locations are detected by the depth sensor which serves to identify the flat surface and the aggregates of points. Then the color camera records the image, the virtual models of potential objects are selected for the areas predetermined by the depth sensor, the appropriate points for grasping are identified and a part of them are used for grasping the object.

The process of correlating the objects observed by the computer vision system represents a series of the operations with the image as follows:

- Distinguishing static objects from the background 6-38

- Calculating special points in the image and building the descriptors invariable relative to the rotations of the image for the set of special points $59-42$

- Identifying the interest area in the image (object contour, bounding box 43,44

The application areas of the contour analysis are restricted by some certain peculiar features basically associated with the problems of identifying the contour in the images:

- Due to the same brightness the boundary between the object and the background can be indistinguishable, or there may be interferences that make it impossible 
to identify the contour;

- Objects that overlap each other or are regrouped can lead to incorrect identification of the contour and thus the contour does not outline the borders of the object properly.

However, considering just the contours of the objects makes it possible to move from the space of the image to the space of the contours which decreases the complexity of the computational algorithms considerably. Thus, contour analysis possesses low resistance against interference and any crossings or partial visibility of the objects may result in the impossibility of detection or in false identifications; however the simplicity and processing speed of the contour analysis make it possible to apply this approach quite successfully.

- Calculating the spatial coordinates of the points that belong to the object

The information about the spatial coordinates can be obtained by means of employing the depth sensors (for example, Microsoft Kinect) that represent 3D scanners of the environment. The coordinates of the objects are linked to the system of coordinates that is connected with the optical center of the sensor.

- Object approximation with primitive geometric shapes (orb, plane, cube, parallelepiped)

When the knowledge is recovered (skills are formed) in the process of analyzing the data of the video system for the purposes of correlating the objects with the relevant operations the task of object identification can be solved using special algorithms for extracting special points from the image (AKAZE, SUFT, BREAK, SIFT) followed by their comparison with the known templates and also using the tools of artificial neural networks.

To improve the quality of identifying separate objects when knowledge is recovered in the process of skill learning it is possible to apply the approach founded on processing the data of $3 \mathrm{D}$ computer vision system which, apart from the data on color (intensity) of the object, also uses the data on the distance to the optical system of the camera; in this case the task of the image segmentation into standalone objects applies such new criterion as distance. Thereat, the task of object classification can be solved by means of estimating the degree of proximity of $3 \mathrm{D}$ models of the object to some set of primitive geometric shapes (orb, cube, cylinder, taper, pyramid).

Thus, collaborative application of the methods of processing $3 \mathrm{D}$ images and the methods of the contour analysis is considered to be most promising for solving the tasks of object identification and recognition.

\section{Discussion}

Learning methods used for programming movements of manipulators collaborative robots can be presented as Table 1. Specifics of the suggested solution are also represented by collecting the database of the operations performed by manipulator robots which makes it possible not only to improve the efficiency of operations with the earlier studied objects due to shorter time and better computing performance but also to decrease the overall level of energy consumption and to apply the so-called obtained skills to other robots. Thus, it becomes possible to train a group of robots in different operations separately and then to establish a common knowledge base. This approach enables redistributing the tasks of training the robots in new skills between multiple groups of scientists and private users.

It seems advisable that a base of the primitive skills should be developed using which the robot will be able to create the complex operational procedures autonomously in the current environment context building them as though from "bricks". Thereat, the process of learning can be implemented by the examples of the complex tasks when in the dialogue mode the operator would specify the next operation for the robot thus forming the base of skills that could be used by other robots including those of different kinematic design. This will be ensured because the skills are not final movement trajectories but some simplest object directed actions in the context of the environment. Thus, the robot will be capable of performing operations with the objects taking into account the context and indeed building the chain from the basic set of actions in such a way that the most complex task could be fulfilled.

The specific features of using the intellectual system of skills that enables programming the robot for a definite task by way of combining several elementary actions ("bricks" of skills) are as follows:

- Identifying the spatial orientation and the location of the object;

Distinguishing static object and the background;

Approximation of the object outlines using primitive geometric shapes;

Identifying planes for grasping;

- Grasping the object;

Solving reverse kinematic problem (by either 
Table 1. Comparative analysis of manipulator robot programming methods

\begin{tabular}{|c|c|c|c|c|}
\hline No. & Parameter & Traditional & Kinesthetic & Observation-based learning \\
\hline 1 & $\begin{array}{l}\text { Complexity of } \\
\text { programming }\end{array}$ & Highly complex & Simple & Simple \\
\hline 2 & $\begin{array}{l}\text { Possibility to edit } \\
\text { the program }\end{array}$ & No & Yes & \\
\hline 3 & Copying "skills" & \multicolumn{2}{|c|}{$\begin{array}{l}\text { It is possible only under the conditions when } \\
\text { the configurations of the effective areas of dif- } \\
\text { ferent robots are in full conformity with each } \\
\text { other, as the robot does not "understand" the } \\
\text { context of the environment }\end{array}$} & Yes \\
\hline 4 & $\begin{array}{l}\text { Automated } \\
\text { trajectory } \\
\text { formation }\end{array}$ & No & $\begin{array}{l}\text { Partially; obstacles } \\
\text { can be avoided if } \\
\text { the computer vision } \\
\text { option is available }\end{array}$ & $\begin{array}{l}\text { Yes, taking into account } \\
\text { dynamic and static obstacles }\end{array}$ \\
\hline \multirow[t]{3}{*}{4} & \multirow[t]{3}{*}{ Personnel safety } & \multirow[t]{3}{*}{ No } & \multicolumn{2}{|c|}{ Manipulator speed control for different zones of the effective area } \\
\hline & & & \multicolumn{2}{|c|}{ Shock load control by means of torque sensors } \\
\hline & & & & $\begin{array}{l}\text { Preventing unwanted contact interactions or } \\
\text { full stop if safe trajectory cannot be calculated } \\
\text { Switching the safety mode to follow the external } \\
\text { force in demonstration-based programming }\end{array}$ \\
\hline 6 & $\begin{array}{l}\text { Programming } \\
\text { method }\end{array}$ & $\begin{array}{l}\text { Formation of the } \\
\text { intermediate points of } \\
\text { the trajectory by means } \\
\text { of specialized software } \\
\text { (CAM packages) } \\
\end{array}$ & $\begin{array}{l}\text { Physically affecting } \\
\text { the units of the robot } \\
\text { to move in the given } \\
\text { direction }\end{array}$ & $\begin{array}{l}\text { Demonstrating the object and specifying the final } \\
\text { position for relocation }\end{array}$ \\
\hline 7 & $\begin{array}{l}\text { Movement } \\
\text { trajectory control }\end{array}$ & Full & Full & $\begin{array}{l}\text { Trajectory is calculated } \\
\text { automatically based on reverse kinematics and } \\
\text { collision avoidance Trajectory can be specified } \\
\text { through kinesthetic learning }\end{array}$ \\
\hline 8 & $\begin{array}{l}\text { Trajectory } \\
\text { specification pre- } \\
\text { cision }\end{array}$ & High & Medium & Medium \\
\hline 9 & Application area & Industrial robots & \multicolumn{2}{|l|}{ Collaborative robots } \\
\hline
\end{tabular}

numerical or analytical methods) in terms of position for the sixth degree of freedom of the robot in the system of coordinates connected with the computer vision system;

Solving reverse kinematic problem for the seventh degree of freedom of the robot (end-effector orientation) in terms of the grasping planes on the surface of the object in the system of coordinates connected with the sixth degree of freedom;

- Measuring weight;

Solving reverse dynamic problem based on the torque sensor readings

- Platform movement and navigation;

Creating the occupied space map (identifying free zones);

Using the methods of Simultaneous Localization and Mapping (SLAM) based on 3D images obtained by the combined computer vision system;

Planning trajectory of the mobile platform movements;
Mobile platform movement control;

- Controlling the positioning of "arms" within the effective area;

Creating the occupied space map (identifying free zones);

Planning the manipulator trajectories avoiding static and dynamic obstacles;

Solving reverse kinematic problem for the manipulator with six degrees of freedom (the seventh, the last one is used for orientation of the end-effector);

- Relocating the object in the effective area (with preset orientation and to the preset location) taking into account the changing environment (static and dynamic obstacles);

- Coordinated operations with two "arms".

Based on the results of the analysis of the technical level of the research studies in the sphere of developing 
the methods for programming the tasks for multilink manipulators and also for human-robot collaboration based on the visual demonstrations it can be maintained that further stages of the investigations should focus on the following:

1) Formation of the action prototype library sufficient for improving the efficiency of robot learning from demonstrations of typical movements;

2) Development of the methods of programming the tasks and human-robot collaboration based on the visual demonstrations followed by imitating the actions of the operator in the process of learning new operations;

3) Combining two methods of training collaborative robots to achieve maximal effect: kinesthetic (the mode when the units of the manipulator follow the externally applied force) and observation-based learning;

4) Formation of the knowledge database founded on correlating the observed objects with the actions performed with them by applying visual demonstrations when the operations are split into separate fragments and elements of the movements;

5) Using additional programming interfaces for the purposes of learning.

\section{Conclusion}

Specific characteristics of collaborative robots are represented by the fact that the tools for programming or learning are integrated in the robot itself and the programming can be performed directly in the installed robot. There are different methods of implementing the robot training aids: joysticks on the manipulators, torque sensor systems in the joints, virtual reality gloves, etc. The efficiency of the above mentioned aids should be analyzed further; however, it has to be noted, that the analysis will be affected by the subjective factors associated with the ease of use for that or another operator.

Thus, the objects of further investigations in the sphere of developing the methods and tools for programming and training collaborative robots should cover the development of the universal model of control over performing one particular acquired practice (complex operation with objects) in undetermined environment as a combination of elementary actions performed in realtime mode revealing the capability of adapting to the changing conditions of the environment.

To build the mathematical model of the universal control system the mathematical and software models should be developed describing the kinematics and dynamics of the multilink manipulator using linear and nonlinear differential equations. The software model should be verified for its efficiency, precision and adequacy. In order to undertake the experimental investigations of the developed universal control model the methods for its software implementation should be found which would make it possible to investigate the possibility of adequate coordinated operations of the elements of skills in real-time mode from the perspectives of the efficient skills performance.

Based on the results of the analysis the method for programming the tasks for collaborative robots will be developed.

The method will be founded on two approaches as follows:

a) The manipulator joints should follow the externally applied forces upon processing the data obtained from the torque sensors to determine the directions of the external forces and to follow the specified trajectory automatically;

b) Knowledge recovery based on correlating the operations with the types of the objects and with the reference points on the surface; formation of the database by recognizing the objects based on their images in the computer vision system and in the 3D coordinate identification system.

The method employs the operator's tagging of the target operation areas that corresponds to this particular object. The method is founded on using auxiliary control tools installed on the manipulator end-effector.

In the process of the experimental investigations the manipulator primitive movement library will be developed. This library will represent a typical set of manipulator trajectories and the set of the objects that have been used for the robot skills learning. The database will predetermine the format for representing the skills of the robot and would be used for exchanging the skills between several robots to ensure automatic duplication of the learned operations with the known set of objects and its adaptation to the changing operating conditions (relocating the robot to another place, changing configuration of the effective area, dynamically changing environment).

In order to develop the methods of task programming during the next stages of the study the investigations will have to be performed in the area of solving reverse kinematic problem for multilink manipulator as well as the investigations of the typical operations of the collaborative robots and of the format for their representation in the database, developing the format for storing the "skills" for further use and the investigations of the methods of image recognition to meet the system specifications. 


\section{Acknowledgments}

This research study was financially supported by the Ministry of Education and Science of the Russian Federation under Grant agreement No.14.577.21.0191 dd. October, 27, 2015 (Unique identifier of the agreement: RFMEFI57715X0191); the grant is provided to perform the applied research studies on the subject: "Investigating scientific and technical solutions and developing an experimental prototype of a multi-purpose two-armed robot assistant on a mobile platform that can replace human workers in various remotely performed tasks". The work on the project is carried out at the Moscow State University of Mechanical Engineering (MAMI).

\section{References}

1. RB 14000 YUMI. ABB Ltd [Internet]. 2016 [cited 2016 May 7]. Available from: http://new.abb.com/products/robotics/ industrial-robots/yumi.

2. Kirgis FP, Katsos P, Kohlmaier M. Collaborative robotics in robotic fabrication in architecture, Springer International Publishing. Art and Design; 2016. p. 448-53.

3. Collaborative robot CR-35iA. FANUC Corporation [Internet]. 2016 [cited 2016 May 4]. Available from: http://www.fanuc.eu/pt/en/robots/robot-filter-page/collaborative-cr35ia.

4. LBR iiwa 7 R800. KUKA Roboter GmbH. [Internet]. 2016 [cited 2016 Jul 8]. Available from: http://www.kuka-robotics.com/ en/products/industrial_robots/sensitiv/lbr_iiwa_7_r800.

5. Motoman HC10 collaborative robot - Safe and flexible interaction. YASKAWA Corporation [Internet]. 2016 [cited 2016 Jun 28]. Available from: https://www.yaskawa.eu.com/de/ news-events/news/artikel/news/motoman-hc10-collaborative-robot-safe-and-flexible-interaction/?tx_news_pi $1 \% 5 \mathrm{~B}$ controller\%5D=News\&tx_news_pi $1 \% 5 B$ action $\% 5 \mathrm{D}=$ detail\&cHash=e91562b0cf2f02dac3884111cfacf6dc.

6. ROS. About ROS [Internet]. 2016 [cited 2016 May 10]. Available from: http://www.ros.org/about-ros/.

7. Chitta S, Sucan I, Cousins S. ROS Topics. In the Proceedings of Institute of Electrical and Electronics Engineers (IEEE) Robotics and Automation Magazine. 2012; 19(1):18-9.

8. Foote T, Cousins S. Precisely groovy [ROS Topics]. Institute of Electrical and Electronics Engineers (IEEE) Robotics and Automation Magazine. 2013; 20(1):13-5.

9. Amir MGE, Paxton C, Hager GD, Bascetta, L. An incremental approach to learning generalizable robot tasks from human demonstration. In the Proceedings of Institute of Electrical and Electronics Engineers (IEEE) International Conference on Robotics and Automation (ICRA); 2015 May 26-30. p. 5616-21.

10. Cheng L, Sun Q, Su H, Cong Y, Zhao S. Design and implementation of human-robot interactive demonstration system based on Kinect. In the 24th Chinese Control and Decision Conference (CCDC), Institute of Electrical and
Electronics Engineers (IEEE); 2012 May. p. 971-5.

11. Rybski PE, Yoon K, Stolarz J, Veloso MM. Interactive robot task training through dialog and demonstration. In the 2nd Association for Computing Machinery (ACM)/ Institute of Electrical and Electronics Engineers (IEEE) International Conference on Human-Robot Interaction (HRI); $2007 \mathrm{Mar}$ 9-11. p. 49-56.

12. Liu P, Glas DF, Kanda T, Ishiguro H, Hagita N. How to train your robot-teaching service robots to reproduce human social behavior. In the 23rd International Symposium on Robot and Human Interactive Communication, Institute of Electrical and Electronics Engineers (IEEE); 2014 Aug. p. 961-8.

13. Zhang D, Ikeura R, Mori Y. Motion reproduction by human demonstration based on discrete hidden Markov model for nursing-care assistant robot. In the International Conference on Systems, Man, and Cybernetics (SMC), Institute of Electrical and Electronics Engineers (IEEE); 2014. p. 842-6.

14. Hoyos J, Prieto F, Peña C, Morales E, Perez-Cisneros M. Reaching new positions using an extreme learning machine in programming by demonstration. In Latin American Robotics Symposium and Competition (LARS/LARC), Institute of Electrical and Electronics Engineers (IEEE); 2013 Oct 21-27. p. 100-105.

15. Balakirsky S, Kootbally Z, Kramer T, Pietromartire A, Schlenoff C, Gupta S. Knowledge driven robotics for kitting applications. Robotics and Autonomous Systems. 2013; 61(11):1205-14.

16. Crick C, Osentoski S, Jay G, Jenkins OC. Human and robot perception in large-scale learning from demonstration. In Proceedings of the 6th international conference on human-robot interaction, Association for Computing Machinery (ACM); 2011 March. p. 339-46.

17. Butterfield J, Osentoski S, Jay G, Jenkins OC. Learning from demonstration using a multi-valued function regressor for time-series data. In 10th IEEE-RAS International Conference on Humanoid Robots, Institute of Electrical and Electronics Engineers (IEEE); 2010. p. 328-33.

18. Tan H, Kawamura K. A computational framework for integrating robotic exploration and human demonstration in imitation learning. In the IEEE International Conference on Systems, Man, and Cybernetics (SMC), Institute of Electrical and Electronics Engineers (IEEE); 2011. p. 2501-6.

19. Hulin T, Schmirgel V, Yechiam E, Zimmermann UE, Preusche C, Pöhler G. Evaluating exemplary training accelerators for programming-by-demonstration. In 19th International Symposium in Robot and Human Interactive Communication, Institute of Electrical and Electronics Engineers (IEEE); 2010 Sep. p. 440-5.

20. Pastor P, Kalakrishnan M, Chitta S, Theodorou E, Schaal S. Skill learning and task outcome prediction for manipulation. In the International Conference on Robotics and Automation (ICRA), Institute of Electrical and Electronics Engineers (IEEE); 2011. p. 3828-34.

21. Marzinotto A, Colledanchise M, Smith C, Ögren P. Towards a unified behavior trees framework for robot control. In the International Conference on Robotics and Automation (ICRA), Institute of Electrical and Electronics Engineers 
(IEEE); 2014. p. 5420-7.

22. Bentivegna DC, Atkeson CG, Cheng G. Learning tasks from observation and practice. Robotics and Autonomous Systems. 2004; 47(2):163-9.

23. Niekum S, Osentoski S, Konidaris G, Barto AG. Learning and generalization of complex tasks from unstructured demonstrations. In the International Conference on Intelligent Robots and Systems, Institute of Electrical and Electronics Engineers (IEEE); 2012. p. 5239-46.

24. Konidaris G, Kuindersma S, Grupen R, Barto A. Robot learning from demonstration by constructing skill trees. The International Journal of Robotics Research. 2012; 31(3):360-75.

25. Tenorth M, Beetz M. Know Rob: A knowledge processing infrastructure for cognition-enabled robots. The International Journal of Robotics Research. 2013; 32(5):566-90.

26. Lin HI, Lai CC. Learning collision-free reaching skill from primitives. In the International Conference on Intelligent Robots and Systems, Institute of Electrical and Electronics Engineers (IEEE); 2012. p. 2383-8.

27. Lin HI. Skill learning for assistive robotics. In the Proceedings of International Symposium on System Integration (SICE) Annual Conference, Institute of Electrical and Electronics Engineers (IEEE); 2010. p. 1904-8.

28. Andersen RS, Nalpantidis L, Krüger V, Madsen O, Moeslund TB. Using robot skills for flexible reprogramming of pick operations in industrial scenarios. In the International Conference on Computer Vision Theory and Applications (VISAPP), Institute of Electrical and Electronics Engineers (IEEE). 2014; 3:678-85.

29. Abdo N, Spinello L, Burgard W, Stachniss C. Inferring what to imitate in manipulation actions by using a recommended system. In the International Conference on Robotics and Automation (ICRA), Institute of Electrical and Electronics Engineers (IEEE); 2014. p. 1203-8.

30. Colledanchise M, Ögren P. How behavior trees modularize robustness and safety in hybrid systems. In the International Conference on Intelligent Robots and Systems, Institute of Electrical and Electronics Engineers (IEEE); 2014. p. 1482-8.

31. Vasiliev IA, Popov AV. Control algorithms of a mobile robot with castor wheels in field with barriers. International Journal of Science and Technology. 2015 Nov; 8(29):1-4.

32. Murikipudi A, Prakash, Vigneswaran T. Performance analysis of real time operating system with general purpose operating system for mobile robotic system. International Journal of Science and Technology. 2015 Aug; 8(19):1-6.
33. Suay HB, Toris R, Chernova S. A practical comparison of three robot learning from demonstration algorithm. International Journal of Social Robotics. 2012; 4(4):319-30.

34. Rozo Castañeda L, Calinon S, Caldwell D, Jimenez Schlegl P, Torras C. Learning collaborative impedance-based robot behaviors. In the Proceedings of the Twenty-Seventh Association for the Advancement of Artificial Intelligence (AAAI) Conference on Artificial Intelligence; 2013. p. 1422-8.

35. Yu CC, Wang CC. Interactive learning from demonstration with a multilevel mechanism for collision-free navigation in dynamic environments. In the Conference on Technologies and Applications of Artificial Intelligence, Institute of Electrical and Electronics Engineers (IEEE); 2013. p. 240-5.

36. Nikhil RP, Sankar KP. A review on image segmentation techniques. Pattern recognition. 1993; 26(9):1277-94.

37. Pong PKTK, Bowden R. An improved adaptive background mixture model for real-time tracking with shadow detection. Video-based surveillance systems. Springer US; 2002. p. $135-44$.

38. Zivkovic Z. "Improved adaptive Gaussian mixture model for background subtraction." In the Proceedings of the 17th International Conference on Pattern Recognition, Institute of Electrical and Electronics Engineers (IEEE); 2004 Aug 26.

39. Pablo FA. Fast explicit diffusion for accelerated features in nonlinear scale spaces. IEEE Transactions on Pattern Analysis and Machine Intelligence. 2011; 34(7):1281-98.

40. Pablo FA, Bartoli A, Davison AJ. KAZE features. European Conference on Computer Vision. Springer Berlin Heidelberg. 2012; 7577:214-27.

41. Bay H, Tuytelaars T, Gool LV. Surf: Speeded up robust features. European conference on computer vision. Springer Berlin Heidelberg. 2006; 3951:404-17.

42. Alexandre A, Ortiz R, Vandergheynst P. Freak: Fast retina keypoint. In the Conference on Computer Vision and Pattern Recognition (CVPR), Institute of Electrical and Electronics Engineers (IEEE); 2012 Jun 16-21. p. 510-7.

43. Pablo $\mathrm{A}$ et al. Contour detection and hierarchical image segmentation. Institute of Electrical and Electronics Engineers (IEEE) Transactions on Pattern Analysis and Machine Intelligence. 2011; 33(5):898-916.

44. Xiaolong D, Khorram S. A feature-based image registration algorithm using improved chain-code representation combined with invariant moments. Institute of Electrical and Electronics Engineers (IEEE) Transactions on Geoscience and Remote Sensing. 1999; 37(5):2351-62. 